\title{
Autoria de Atividades de Aprendizagem Adaptativas com base na Inteligência Coletiva
}

\author{
Alan C. S. Barreto ${ }^{12}$, Eveline J. V. Sá ${ }^{3}$, Omar A. C. Cortes ${ }^{3}$, Dario Vieira ${ }^{4}$ \\ ${ }^{1}$ Universidade Estadual do Maranhão \\ Caixa Postal 09, CEP. 65055-310 - São Luís - MA - Brasil \\ ${ }^{2}$ Instituto Federal de Educação, Ciência e Tecnologia do Pará (IFPA) \\ CEP 68515-000 - Parauapebas - Pará - Brasil \\ ${ }^{3}$ Instituto Federal de Educação, Ciência e Tecnologia do Maranhão (IFMA) \\ Caixa Postal 65030-005 - São Luís - MA - Brasil. \\ ${ }^{4}$ Engineering School of Information and Digital Technologies (EFREI) \\ Villejuif, France
}

alan.barreto@ifpa.edu.br; eveline,omar\{@ifma.edu.br\};dario.vieira@gmail.com

\begin{abstract}
The pre-3AJC process proposed in (Sá, 2011) guides the development of learning activities through the use of games, cooperation, and sequencing learning objects of different types, such as videos, audios, texts, images, etc. However, despite all the flexibility of this pre-authorship process, there is still a limitation regarding the creation of learning activities adaptable to the learner's profile. In this sense, this article presents a prototype authoring tool based on the pre-3AJC process, and an ACO (Ant Colony Optmization) collective intelligence algorithm, to adapt according to the learner profile the contents presented in the developed learning activities.
\end{abstract}

Resumo. O processo de pré-autoria pré-3AJC proposto em (Sá, 2011) orienta o desenvolvimento de atividades de aprendizagem, através do uso de jogos, cooperação, além do sequenciamento de objetos de aprendizagem de diversos tipos, tais como, vídeos, áudios, textos, etc. Entretanto, apesar de toda flexibilidade deste processo de pré-autoria, ainda há limitação referente à criação de atividades de aprendizagem (AA) adaptáveis ao perfil do aprendiz. Neste sentido, este artigo apresenta um protótipo de ferramenta de autoria baseado no processo pré-3AJC e um algoritmo de inteligência coletiva ACO (Ant Colony Optmization) adaptado, para adequar de acordo com o perfil do aprendiz os conteúdos apresentados nas AAs desenvolvidas.

\section{Introdução}

No mundo da tecnologia da informação é possível notar uma grande diversidade de sistemas (e/ou aplicações) com foco na educação, com o intuito de auxiliar o processo de ensino-aprendizagem, tais como: ambientes virtuais de aprendizagem, jogos educativos, ferramentas de autoria, ou simplesmente, uma apresentação de slides no 
ambiente de sala de aula. As ferramentas de autoria destacam-se pela produção de materiais didáticos em diversos formatos pelo professor, possibilitando sua reutilização em momentos posteriores. Segundo Silva (2013), a ferramenta (ou software) de autoria, é um aplicativo equipado com diversas ferramentas de multimídia que, a partir de modelos pré-definidos, possibilita ao usuário inserir informações, imagens e sons, promovendo a autonomia e estimulando o desenvolvimento cognitivo de quem cria atividades a partir dele.

Segundo o W3C (2015), ferramenta de autoria é qualquer aplicativo, parte de um aplicativo ou coleção de aplicativos com os quais o autor interage para produzir, alterar ou montar conteúdo web, que será utilizado por outras pessoas. Nôleto (2014) afirma que as ferramentas de autoria visa atingir um público mais leigo para auxiliar no processo de produção de conteúdo/material, em sua maioria didáticos. Porém, ainda se percebe uma limitação em lidar com as particularidades de cada aprendiz, como por exemplo, prover material didático que possa se adequar ao seu perfil; o que tem sido um grande desafio para os professores/educadores, bem como, para os processos de autoria de materiais didáticos.

Dentro desse contexto, Dorça et al. (2011) apresenta uma abordagem para a identificação e ajuste automático de estilos de aprendizagem (EAs) baseada em cadeias de Markov, a fim de que as plataformas e-learning considerem as características únicas de cada estudante/usuário, permitindo que este possa selecionar caminhos dentro dos mecanismos gerais disponibilizados pelo professor/tutor. Resende et. al., (2014) apresentam uma abordagem para mapeamento de características de EAs no modelo de Felder e Silverman e metadados de OAs no padrão LOM, a fim de recuperar objetos de aprendizagem de acordo com os estilos de aprendizagem de um aprendiz específico.

O processo de pré-autoria pré-3AJC proposto em (Sá, 2011), é baseado no padrão IMS-LD, e orienta o desenvolvimento de materiais didáticos que utilizam jogos e cooperação como recursos educacionais, chamados de atividades de aprendizagem, a partir do uso das melhores práticas do IMS-LD para representar aspectos pedagógicos. Apesar de toda flexibilidade deste processo de pré-autoria, este ainda se encontra limitado no que se refere a criar atividades de aprendizagem adaptáveis ao perfil do aprendiz. Considerando esse contexto, foi utilizado o algoritmo de inteligência coletiva ACO (Ant Colony Optmization) em conjunto com o modelo de Estilo de Aprendizagem de Felder e Silverman como alternativa para adaptar os conteúdos apresentados nas atividades de aprendizagem desenvolvidas de acordo com o referido processo de préautoria.

No próximo tópico serão apresentados a justificativa e relevância. Na Seção 3 explana-se a metodologia utilizada. Na Seção 4, apresentam-se alguns trabalhos relacionados à temática. Na Seção 5, explana-se a integração do ACO adaptado ao processo pré-3AJC, bem como, funcionamento e implementação da ferramenta de autoria adaptativa; por fim, as considerações finais na Seção 6.

\section{Justificativa e Relevância}

Ao iniciar um processo de ensino-aprendizagem, no ensino tradicional, é comum ser adotado pelo professor um único tipo de material a ser disponibilizado para o aprendiz, sendo que os aprendizes não têm as mesmas afinidades com o mesmo material, logo 
parte dos aprendizes podem apresentar dificuldades na assimilação do conteúdo do assunto abordado na Atividade de Aprendizagem. Uma problemática comum no meio acadêmico é a dificuldade de adequar-se às particularidades de cada aprendiz em uma dada Atividade de Aprendizagem de forma que maximize o seu poder de assimilação, e portanto, atinja os Objetivos Instrucionais, esperados pelo professor, de determinado assunto.

Para minimizar esse tipo de problema comum em uma Atividade de Aprendizagem, é importante adequar os Objetos de Aprendizagem ao aprendiz (material didático), de forma que ele consiga assimilar, o máximo possível, do conteúdo seguindo a sua afinidade diante de um Tipo de Objeto. Com isso, tem-se como técnica de aprimoramento, a utilização de Algoritmos de Inteligência Coletiva, de forma que possibilite adaptar uma Atividade de Aprendizagem da melhor maneira possível ao perfil do aprendiz, que irá estudar determinado conteúdo. Sendo assim, será possível tornar a atividade mais agradável ao aprendiz, visando estimular ao máximo o aproveitamento na atividade de aprendizagem, de acordo com seu estilo de aprendizagem.

\section{Metodologia}

A investigação da pesquisa caracterizou-se como um estudo descritivo de abordagem qualitativa e experimental que foi desenvolvido com a realização das seguintes etapas:

a) Levantamento do referencial teórico e revisão de literatura;

b) Analise e especificação do perfil do aprendiz e suas particularidades para definição de uma base de dados e modelagem dos requisitos de adaptabilidade;

c) Estudo e Implementação do Algoritmo de Inteligência Coletiva, especificamente o ACO (Ant Colony Optimization);

d) Extensão do algoritmo ACO adicionando a capacidade de adaptação;

e) Prototipação da ferramenta de autoria segundo o processo pré-3AJC;

f) Integração do ACO adaptado ao protótipo da ferramenta de autoria e testes.

\section{Trabalhos Relacionados}

\subsection{AntStudy: Proposta de definição de sequências de estudo utilizando Ant System}

A proposta de Araújo e Fagundes (2011) é utilizar o padrão LOM e modelos de usuários como entradas para o algoritmo ACO (Ant Colony Optimization), do tipo Ant System, que terá como objetivo definir as sequências de estudo em um ambiente virtual de aprendizagem. O padrão LOM é utilizado como modelo de metadados para caracterizar objeto de aprendizagem, onde pode-se identificar as informações: complexidade, interatividade, sequências, entre outros. O modelo de usuário (MU) especifica os conhecimentos e as habilidades do aprendiz.

\subsection{NextStep: Um Protótipo para o Sequenciamento Inteligente e Adaptativo de Enunciados em Programação de Computadores}

A pesquisa de Moreira et. al, (2016) consiste em propor o sequenciamento adaptativo de enunciados referente ao conteúdo de Programação de Computadores, tomando como base uma modelagem dinâmica do aprendiz. Um protótipo de ferramenta para o sequenciamento foi implementado, chamado de NextStep, que utiliza grafos genéticos 
como base para a representação interna e externa, possibilitando destacar o progresso do aprendiz sobre o conhecimento do domínio. A partir dos modelos do aprendiz e do domínio, é possível definir um processo de ordenação heurística que orienta conteúdos a serem explorados pelo aprendiz. Mas, é necessário que sejam indicados inicialmente um conjunto de pericias relativas ao domínio e a catalogação de enunciados descritos como subgrafos do conhecimento de domínio, para que a ordenação heurística indique quais perícias devem ser prioritariamente desenvolvidas pelo aprendiz para, em seguida, apresentar os enunciados que contemplem essa demanda. O cenário de utilização do NextStep simula e acompanha o processo de sequenciamento de enunciados para um aprendiz, conforme as etapas, a saber: (1) situar o aprendiz; (2) sugerir perícia; (3) sugerir enunciado; e (4) avaliar solução.

\subsection{Modelo de Apresentação Adaptativa de Objeto de Aprendizagem baseada em Estilos de Aprendizagem}

O modelo de apresentação adaptativa de objetos de aprendizagem proposto em Silva et. al, (2016) teve como base as propriedades dos estilos de aprendizagem do modelo de Felder-Silverman (1988), que a partir de um estudo e investigação, pode-se definir as "características de apresentação para OA" em relação a sequenciamento, apresentação e forma/formato de conteúdo e recursos que compõem o OA, dos quais pode-se estabelecer os parâmetros e atributos necessários para definição do modelo.

Segundo o modelo de adaptação, o OA será formado por "elementos de composição de conteúdo" que constituem as seguintes etapas:

- Resumo (Res): fornece uma visão geral e clara do conteúdo a ser abordado;

- Introdução (Int): conteúdo com a breve descrição do assunto a ser abordado;

- Desenvolvimento (Des): conteúdo com descrição mais abrangente do assunto de uma forma mais completa;

- Atividade (Ati): conteúdo para fixação do assunto; e,

- Avaliação (Ava): avaliação do conteúdo abordado.

Os "elementos de composição de conteúdo" listados são organizados em relação aos parâmetros e atributos definidos no modelo de apresentação adaptativa. Estes parâmetros e atributos combinados aos elementos de criação e composição do $\mathrm{OA}$ estabelecem como o OA pode adaptar-se em função de cada EA.

\section{Ferramenta de Autoria de Atividades de Aprendizagem Adaptativas}

\subsection{Processo pré-3AJC}

O processo de pré-autoria de atividades de aprendizagem pré-3AJC possibilita a autoria de uma atividade de aprendizagem com uso de jogos e cooperação por diferentes aprendizes em diferentes etapas desta atividade de aprendizagem, tomando como base um modelo de atividade de aprendizagem com jogo (Sá, 2011).

O professor poderá modelar uma AA-Jogo (Atividade de Aprendizagem com Jogo) formada por subatividades que possibilitam preparar o aprendiz, inferir informações do aprendiz, aquecer, assimilar e avaliar o aprendiz usando o jogo como recurso pedagógico, bem como discutir os resultados dessas subatividades, a fim de verificar o aprendizado alcançado. Uma AA-Jogo é formada pela sequência de 5 partes 
VI Congresso Brasileiro de Informática na Educação (CBIE 2017)

Anais do XXVIII Simpósio Brasileiro de Informática na Educação (SBIE 2017)

estruturais: Inicial, Desenvolvimento/Preliminar, Desenvolvimento/Intermediário, Desenvolvimento/Final e Encerramento, conforme Figura 1.

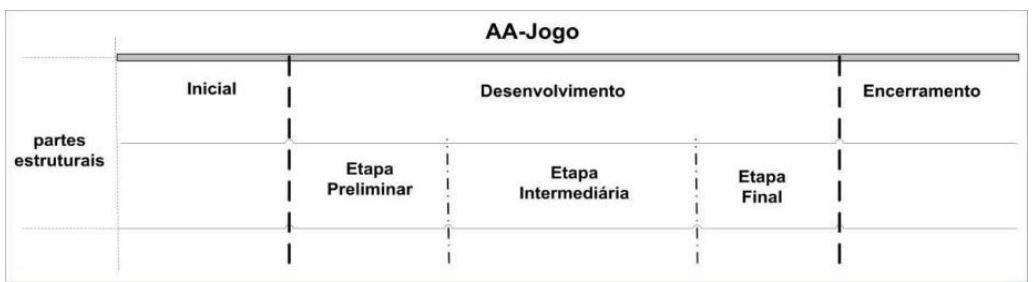

Figura 1. Partes estruturais da AA-Jogo. Fonte: Sá et. al. (2011)

As partes Inicial e Encerramento, são responsáveis por apresentar as informações pertinentes à atividade de aprendizagem (objetivos instrucionais, conteúdo abordado, se usará jogo, se terá avaliação no final da atividade, etc.) e de socializar o fechamento da AA (conclusões relativas ao objetivo instrucional previsto), respectivamente. $\mathrm{Na}$ parte de Desenvolvimento são definidas 3 etapas, que podem ser utilizadas pelo professor sequencialmente ou de forma independente, e são descritas a seguir:

a) Fase Preliminar: É uma fase opcional, porém, sua inclusão pode proporcionar um melhor rendimento na aprendizagem, uma vez que, as subatividades realizadas podem servir como feedback ao professor com informações dos aprendizes, bem como para estimular os aprendizes no início da realização da AA, chamadas de Atividades de Aquecimento (AAq) submetidas aos aprendizes de forma coletiva ou individual.

b) Fase Intermediária: fase onde se encontra a AA propriamente dita. São trabalhadas atividades que desenvolvem competências e habilidades nos aprendizes, relacionadas à aquisição de conhecimento sobre o conteúdo tratado pela AA. Podem ser inseridos quantos objetos de aprendizagem e jogos forem necessários, de acordo com os objetivos instrucionais definidos.

c) Fase Final: fase onde são aplicados nos aprendizes subatividades para inferir o conhecimento do aprendiz referente ao conteúdo trabalhado, bem como, avaliar se as habilidades e competências foram alcançadas.

Dentre algumas limitações do Processo pré-3AJC, foi detectada ausência da capacidade de adaptação em relação ao perfil do aprendiz, podendo notar que a escolha do jogo, bem como dos OAs, é definido pelo professor. Portanto, a adaptabilidade poderia ser uma forma de otimizar o processo da atividade de aprendizagem, levando em consideração o perfil do aprendiz, como é o caso dessa proposta. No entanto, não será contemplado o jogo como um recurso didático, uma vez que estes possuem características mais especificas que os objetos de aprendizagem. Primeiramente, a adaptação de conteúdos de acordo com o perfil, será aplicada apenas aos objetos de aprendizagem (do tipo: slide, vídeo, áudio, texto, etc.), posteriormente, estendida para jogos.

\subsection{ACO}

O Ant Colony Optimization (ACO) é uma meta-heurística baseada no comportamento de colônias de formigas em busca de comida. As formigas se comunicam através de um feromônio, uma substancia química, utilizada para criar as trilhas que orientam até a comida. A partir do feromônio deixado pelas formigas, as demais formigas seguem essa 
VI Congresso Brasileiro de Informática na Educação (CBIE 2017)

Anais do XXVIII Simpósio Brasileiro de Informática na Educação (SBIE 2017)

trilha, depositando também feromônios, aumentando assim a probabilidade que este caminho seja utilizado. A primeira versão do ACO conhecida como Ant System (AS) definida por (Dorigo et al, 1991) foi aplicado a problemas de otimização combinatória em domínios discretos, como por exemplo, caixeiro viajante, roteamento, etc.

Observa-se no algoritmo ACO adaptado que foi necessária a definição de novos parâmetros: estilo de aprendizagem do aprendiz (EA_AP); estilo de aprendizagem do objeto de aprendizagem (EA_OA), tipo do objeto; assim como, a busca pelo melhor caminho, que colabora na escolha de quais OAs se adequam melhor ao aprendiz de acordo com seu estilo de aprendizagem. $\mathrm{O}$ algoritmo de Otimização de Colônia de Formigas (ACO) adaptado é apresentado a seguir:

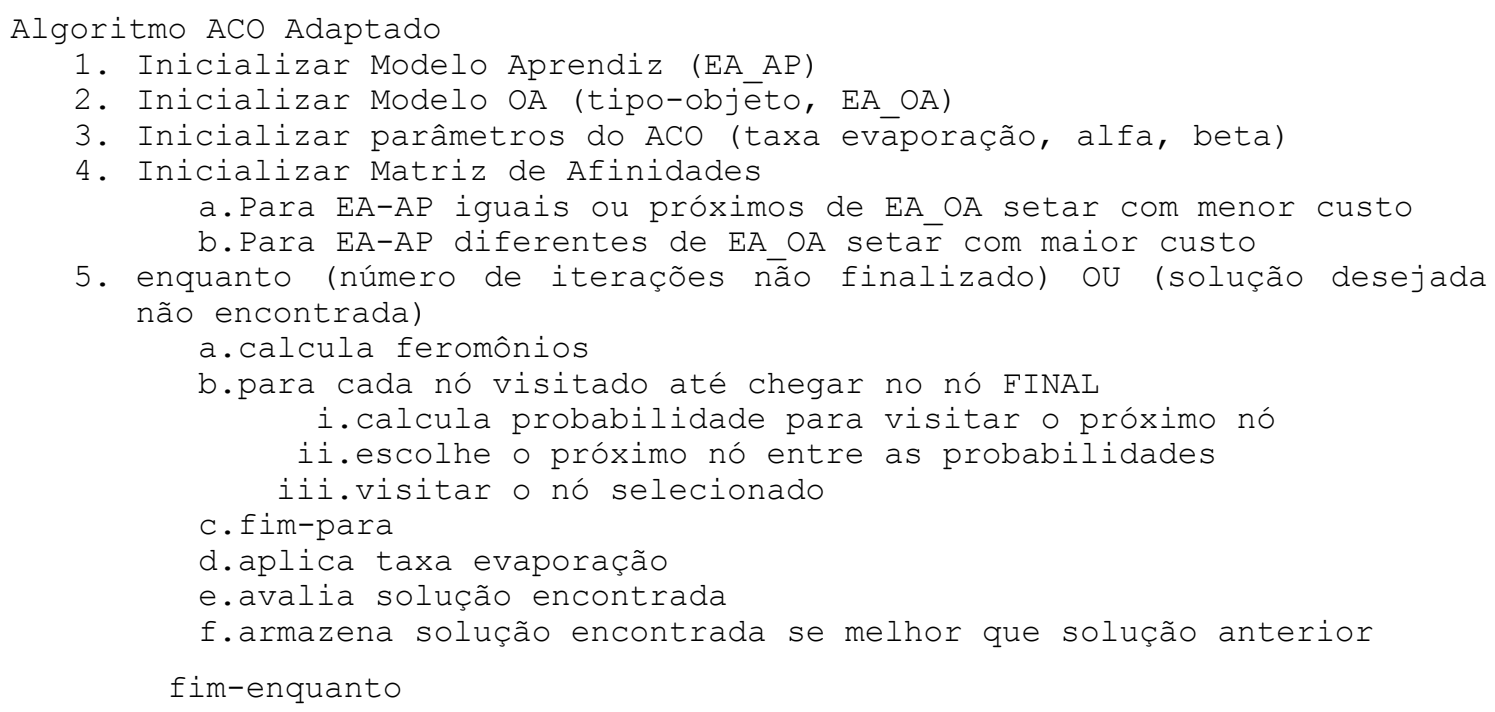

O estilo de aprendizagem considerado será o definido por Felder e Silverman (1988), que o caracteriza em 4 dimensões, a saber: ativo-reflexivo, sensitivo-intuitivo, visual-verbal, sequencial-global. A função de avaliação de cada nó (solução local), é dada pela probabilidade de cada nó, definida pela seguinte função matemática, e após o cálculo, é escolhido o nó de maior probabilidade (método de seleção é guloso, ou seja, o melhor no momento).

$$
\text { Probabilidade }=\frac{\text { feromônio }^{\alpha} \cdot\left(\frac{1}{\text { distancia }}\right)^{\beta}}{\text { somatorio }} \quad \begin{gathered}
\text { se somatorio }>0 \\
\text { senao, somatorio igual a } 1
\end{gathered}
$$

Após obter uma solução completa (ter selecionado todos os nós), a solução é avaliada comparando com a \% (porcentagem) de objetos que se adequam ao perfil do aprendiz (EA), onde o que apresentar maior que $80 \%$, é considerado um excelente roteiro de objetos para atividade de aprendizagem, além de ser um dos critérios de parada de execução do ACO adaptado.

\subsection{Integração do ACO ao processo pré-3AJC}

Inicialmente foi necessário especificar os requisitos da Ferramenta de Autoria de Atividades de Aprendizagem segundo processo pré-3AJC, ilustrados nos diagramas de casos de usos apresentados na Figura 2. Observa-se, que existem dois atores: o professor e o aprendiz, que por sua vez, definiram os Módulos Professor e Aprendiz. 

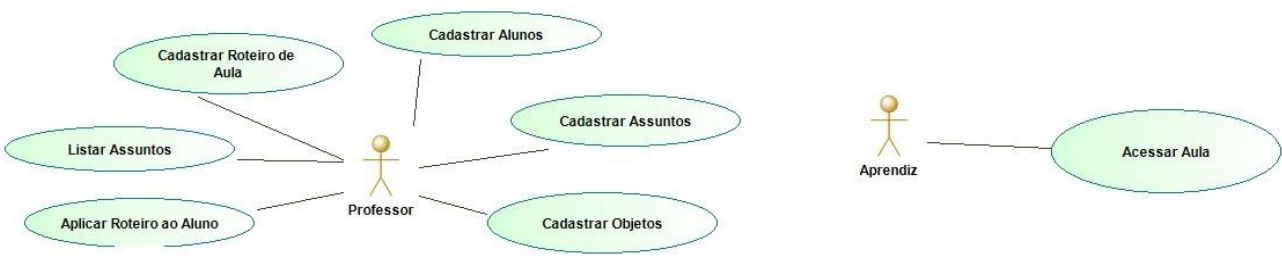

\section{Figura 2. Casos de Usos Ferramenta de Autoria de Atividades de Aprendizagem Adaptativas}

A estrutura da atividade de aprendizagem no processo pré-3AJC se manteve as suas fases e etapas, conforme figura 1, mesmo com a inserção do ACO adaptado apresentado na seção 5.2. Correspondente a cada uma dessas fases e etapas, ilustradas na Figura 3, tem-se duas visões da ferramenta, considerando o Diagrama de Casos de Uso apresentado na figura 2: Ferramenta de Autoria/Módulo Professor e Ferramenta de Autoria/Módulo Aprendiz.

Como pode ser visto na Figura 3, as fases Inicial e Encerramento, e etapas Preliminar/Aquecimento e Final/Avaliação do processo pré-3AJC correspondem às mesmas fases e etapas na Ferramenta de Autoria em ambos os Módulos Professor e Aprendiz, respeitando as funcionalidades dos casos de uso Cadastrar Roteiro de Aula e Acessar Aula, respectivamente.

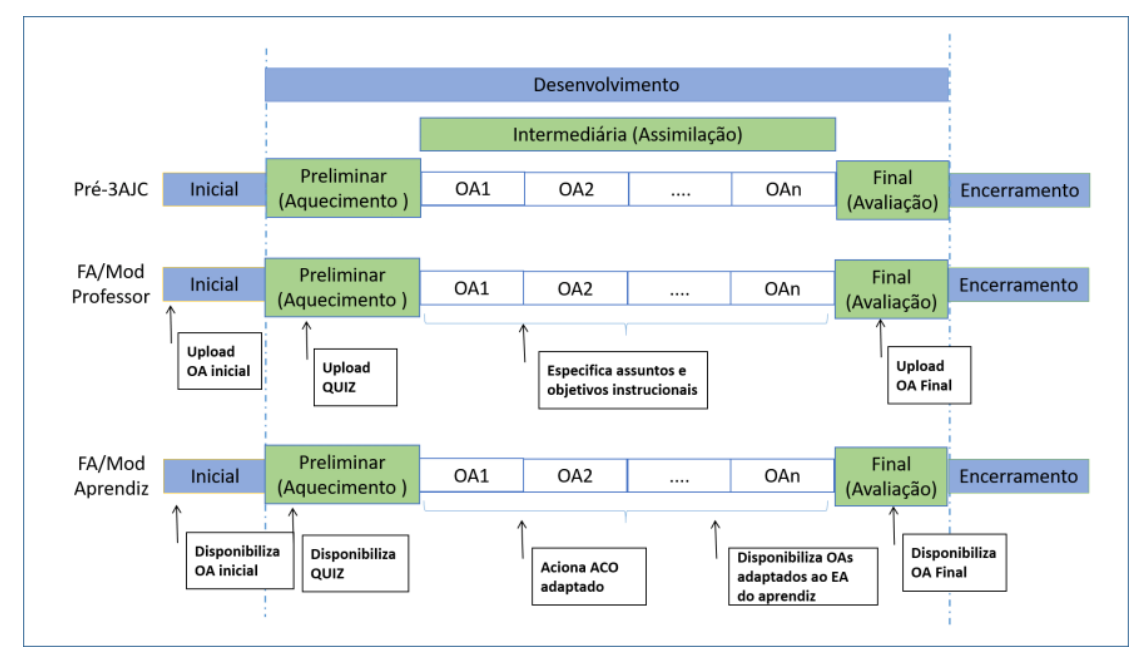

Figura 3. Integrando ACO ao processo pré-3AJC

A etapa de Preliminar/Aquecimento, no processo pré-3AJC prevê o uso de jogo como recurso para essa dinâmica. No contexto atual, será aplicado um "Pergunta e Resposta" em formato de "QUIZ" em substituição ao jogo, para sondar o nível de conhecimento (NC) do aprendiz nos assuntos a serem abordados pelo atividade de aprendizagem, uma vez que não faz parte do contexto desta pesquisa considerar o jogo como objeto de aprendizagem. O ACO será acionado quando o aprendiz estiver acessando a aula (caso de uso Acessar aula) que já teve sua autoria realizada pelo professor, através do caso de uso Criar Roteiro de Aula. 
VI Congresso Brasileiro de Informática na Educação (CBIE 2017)

Anais do XXVIII Simpósio Brasileiro de Informática na Educação (SBIE 2017)

\subsection{Prototipação da Ferramenta de Autoria de Atividades de Aprendizagem Adaptativa}

Para o desenvolvimento do protótipo da Ferramenta de Autoria, utilizou-se como tecnologias para o desenvolvimento: a Linguagem de Programação Python, para a implementação do ACO; o Framework Django (ferramenta baseada no Python), para a implementação do protótipo Ferramenta de Autoria, possibilitando que está possa ser acessível via web, o banco de dados com o PostgreSQL, para o armazenamento das informações necessárias, tais como: o perfil do Aprendiz, os Roteiros de Atividades de Aprendizagem, dentre outras informações.

A Figura 4 ilustra uma funcionalidade da Ferramenta de Autoria referente ao caso de uso Cadastrar Roteiro Aula, onde o professor especifica os assuntos a serem abordados em cada etapa da AA, bem como as "Perguntas e Respostas" que serão aplicados ao aprendiz, a fim de sondar o seu respectivo NC. Para compor o "Perguntas e Respostas" serão usadas duas questões por assunto abordado na AA.

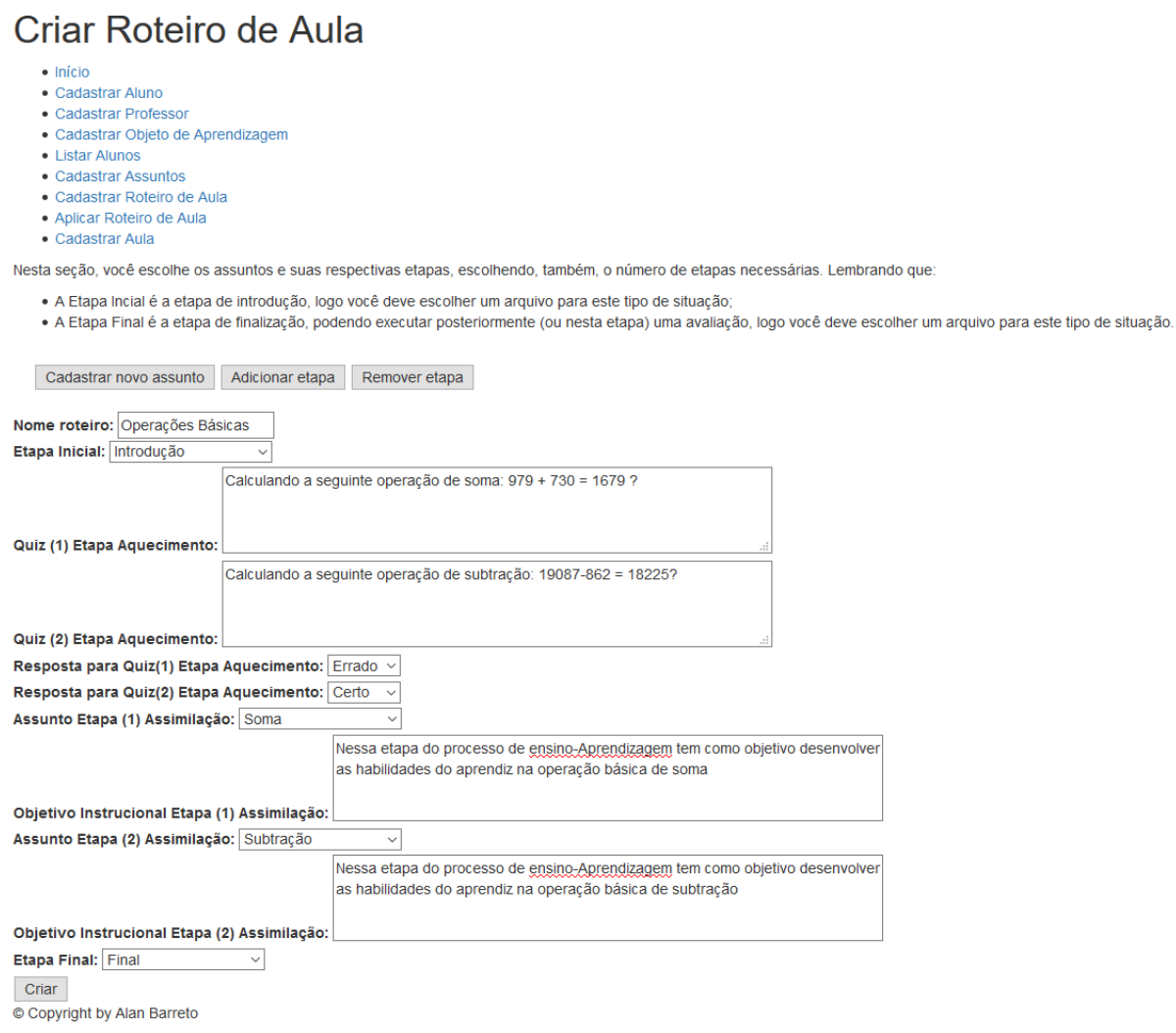

Figura 4. Tela para Criar Roteiro de Aula

De acordo com o resultado de acertos será extraído o $\mathrm{NC}$, que determinará se é necessário ou não, a apresentação de um $\mathrm{OA}$ extra como reforço para aquele assunto naquela AA. Posteriormente com essas informações, o ACO buscará os OAs desses assuntos e aplicará na etapa específica. A funcionalidade da Ferramenta de Autoria Acessar Aula, do módulo do Aprendiz, é subdividida em duas partes. A primeira, o aprendiz tem acesso as atividades de aprendizagem (aulas) que estão disponíveis para ele, como ilustrado na Figura 5. 


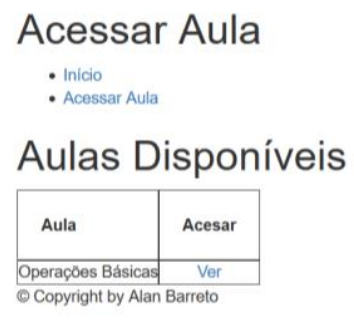

Figura 5. Tela para Acessar Aula. Fonte: Autor (2017).

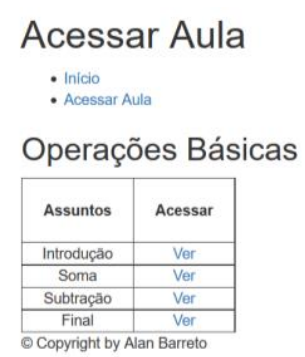

Figura 6. Tela para Acessar Assuntos da Aula. Fonte: Autor (2017).

No exemplo da Figura 5, a aula disponıveı e sobre uperaçoes basıcas, do conteúdo de Matemática. A segunda parte, o aprendiz tem acesso aos assuntos relacionados ao roteiro de aula criado para o conteúdo "Operações Básicas", que no exemplo, são: Introdução, Soma, Subtração e Divisão, seguido do link para acessar os OAs que serão definidos pelo ACO para cada assunto de acordo o tipo do objeto e EA do aprendiz, conforme ilustrado pela Figura 6.

\section{Considerações Finais}

A proposta deste artigo inserir a capacidade adaptativa ao processo pré-3AJC (Sá, 2011) na autoria de AA que possam ser adaptáveis de acordo com o perfil do aprendiz, bem como, o protótipo da ferramenta de autoria do referido processo. Como técnica para prover tal adaptação, investigou-se o uso do algoritmo de inteligência coletiva ACO (Ant Colony Optmization), que foi adaptado para receber as informações do aprendiz (estilo de aprendizagem) e informações dos objetos de aprendizagem (tipo do objeto).

Nos testes iniciais, com o ACO adaptado para atender o cenário de utilização da ferramenta de autoria, foi possível notar, considerando 6 etapas (incluindo as etapas inicial e final), um grafo dirigido com 17 nós, uma formiga e depois duas formigas, obteve-se um roteiro de acordo com o peso de afinidade entre os tipos de objeto e o EA do aprendiz. O menor peso se refere ao objeto que o aprendiz possui maior afinidade. $\mathrm{O}$ método de seleção de nós para as formigas no teste, considera o método guloso, decide pela melhor opção naquele momento, ou seja, buscando o melhor nó na etapa especificada (busca local), e como parâmetro de escolha, usa a probabilidade, onde a maior probabilidade se refere ao melhor nó para escolha. A convergência tende a chegar rapidamente, tanto com uma ou duas formigas, devido ao número baixo de nós, do método de seleção (guloso), e do grafo ser dirigido, o que demanda (inicialmente) pouco processamento. $\mathrm{O}$ resultado do algoritmo é a porcentagem de objetos referente as afinidades do aprendiz em relação ao tipo de objeto, onde quanto maior a porcentagem, melhor o roteiro obtido pelo ACO adaptado, sendo esse critério de parada caso atinja $80 \%$ ou mais. Como trabalhos futuros, está previsto integrar o ACO adaptado ao protótipo da ferramenta de autoria para realizar os testes com dados em tempo de execução.

\section{Agradecimentos}

A FAPEMA, pelo fomento à pesquisa. 
VI Congresso Brasileiro de Informática na Educação (CBIE 2017)

Anais do XXVIII Simpósio Brasileiro de Informática na Educação (SBIE 2017)

\section{Referências}

Araújo, Lucas Moreno; Fagundes, Fabiano. (2011) AntStudy: Proposta de definição de sequências de estudo utilizando Ant System. In: ENCONTRO DE COMPUTAÇÃO E INFORMÁTICA DO TOCANTINS, 13., 2011, Palmas. Anais... Palmas: CEULP/ULBRA. p. 98-108.

Dorça, Fabiano Azevedo; Lima, Luciano Vieira; Fernandes, Márcia Aparecida; Lopes, Carlos Roberto. (2011). Detecção e Correção Automática de Estilos de Aprendizagem em Sistemas Adaptativos para Educação. Disponível em: <http://www.seer.ufrgs.br/rita/article/view/rita_v18_n2_p178>. Acesso em: julho/17.

Dorigo, M.; Maniezzo, V.; Colorni, A. (1991). Positive feedback as a search strategy. Dipartimento di Elettronica, Politecnico di Milano, Italy, Technical Report TR 91016

Felder, R., Silverman, L. (1988). Learning and teaching styles in engineering education. Journal of Enginee ring education, 78(7), 674-681.

Moreira, Carolina; Pimentel, Andrey Ricardo e Maschio, Eleandro. (2016). NextStep: Um Prototipo para o Sequenciamento Inteligente e Adaptativo de Enunciados em Programação de Computadores. Anais do XXVII Simpósio Brasileiro de Informática na Educação (SBIE 2016) . V CBIE 2016. Uberlândia - MG.

Nôleto, Carleandro O.; Viana, Windson; Trinta, Fernando A.M.. (2014). Uma Ferramenta de Autoria para o Desenvolvimento de Jogos Pervasivos Baseados em Realidade Aumentada. Disponível em: $<$ http://www.lbd.dcc.ufmg.br/colecoes/wtdwebmedia/2014/005.pdf $>$. Acesso em julho/2017.

Resende, Daniel Teixeira; Dorça, Fabiano Azevedo; Cattelan, Renan G.; Araújo, Rafael D.. (2014). Em direção à recuperação automática de objetos de aprendizagem em repositórios através da associação dos estilos de aprendizagem de estudantes com metadados no padrão IEEE-LOM. CBIE 2014. WCBIE 2014. Dourados - MS.

SÁ, Eveline de Jesus Viana. (2011). Processo de pré-Autoria para Atividades de Aprendizagem com Uso de Jogos e Cooperação. Tese (Doutorado em Engenharia Eletrônica e Computação). ITA, São José dos Campos - SP.

Silva, José Cláudio Moreira da. (2013). O Uso do Software de Autoria JCLIC como Ferramenta Pedagógica no Processo de Ensino-Aprendizagem de Conteúdos da Matemática nas Séries Finais do Ensino Fundamental por Meio da Construção de Jogos Educativos. Dissertação (Mestrado Profissional em Ensino de Ciências e Matemática) Universidade Federal do Ceará, Fortaleza.

Silva, Zenaide Carvalho da; Ferreira, Leandro Rodrigues; e Pimentel, Andrey Ricardo. (2016). Modelo de Apresentação Adaptativa de Objeto de Aprendizagem baseada em Estilos de Aprendizagem. Anais do XXVII Simpósio Brasileiro de Informática na Educação (SBIE 2016). V CBIE 2016. Uberlandia - MG.

W3C. BrowsersandAuthoringTools.Disponível em:

$<$ https://www.w3.org/standards/agents/authoring $>$. Acessado em julho/2017. 